\title{
The Kumaraswamy-Half-Cauchy distribution: properties and applications
}

\author{
Indranil Ghosh \\ Mathematics and Statistics, Austin Peay State University, \\ Clarksville, TN 37044, USA \\ ghoshi@apsu.edu
}

Received 24 December 2013

Accepted 20 February 2014

\begin{abstract}
In this article, based on the half-Cauchy distribution, we propose a new distribution called KumaraswamyHalf-Cauchy distribution. Various explicit expressions for it's moments, generating and quantile functions, mean deviations, reliability parameter, density function of the order statistics and their moments are provided. We consider the method of maximum likelihood to estimate the model parameters. For illustrative purposes, a real life data set is considered as an application of our new distribution.
\end{abstract}

Keywords: Half-Cauchy distribution, Kumaraswamy-Half-Cauchy distribution; Rennyi's entropy; Order statistics.

2000 Mathematics Subject Classification: 22E46, 53C35, 57S20

Keywords and Phrases: Half-Cauchy distribution, Kumaraswamy-Half-Cauchy distribution, Rennyi's entropy, order statistics.

\section{Introduction}

Half-Cauchy distribution (hereafter HC distribution) provides an alternative to inverse-Gamma distribution as a default prior for a scale parameter in Bayesian hierarchical models, in particular, when a proper prior is necessary (Poloon et al., 2011). It is obtained from the standard Cauchy distribution by folding the curve on the origin so that only positive values can be observed. As a heavy tailed distribution, the $\mathrm{HC}$ distribution has been used as an alternative to exponential distribution to model dispensal distances (Shaw, 1995) as the former predicts more frequent long distance dispersed events than the other. The distribution introduced by Kumaraswamy (1980) is quite new among statisticians and has been little explored in the literature. We consider the term "KW" distribution to denote the Kumaraswamy distribution. It's cumulative distribution distribution function (c.d.f.) has a simple form

$$
F(x)=1-\left(1-x^{a}\right)^{b-1}, \quad 0<x<1,
$$


where $a>0$ and $b>0$ are the two shape parameters. The corresponding density function is given by

$$
f(x)=a b x^{a-1}\left(1-x^{a}\right)^{b-1}, \quad 0<x<1,
$$

which can be unimodal, increasing, decreasing or constant, depending on the parameter values. Jones (2009) advocated the KW distribution as a generator since its quantile function takes a simple form. In his paper Jones (2009) highlighted several advantages over beta distribution: simple normalizing constant, simple explicit formula for the distribution and quantile formula for the distribution and quantile functions for the distribution and quantile functions that do not involve any special functions and a simple formula for random variate generation. If $\mathrm{G}$ denotes the c.d.f. of a random variable, Cordeiro and Castro (2011) defined the KW-G distribution given by

$$
F(x)=1-\left(1-G(x)^{a}\right)^{b},
$$

where $a>0$ and $b>0$ are two additional parameters whose role is to introduce skewness and to vary tail weights. Because of its tractable distribution function (1.2), the KW-G distribution can be used quite effectively even if the data are censored. Correspondingly, the density function of this family of distributions has a very simple form

$$
f(x)=\operatorname{abg}(x) G(x)^{a-1}\left(1-G(x)^{a}\right)^{b-1} .
$$

So, the KW-G distribution is obtained by adding two shape parameters $a$ and $b$ to the G distribution. It contains distributions with unimodal and bathtub shaped hazard rate functions. Clearly, the $\mathrm{KW}$ density function in (1.2) is a particular case of (1.4) with $G(x)=x$. In this paper, we combine the works of Kumaraswamy and Cordeiro and Castro (2011) to study mathematical properties of a new distribution referred to as the KW-Half-Cauchy (hereafter KW-HC) distribution. The new model contains a large number of sub-models such as Half-Cauchy, folded t-distribution with degrees of freedom $=1$, among others. The new model is suitable for testing goodness of fit of these sub-models and for defining a regression model. If $X$ follows a Half-Cauchy distribution with parameter $\delta$ with the c.d.f $G_{\delta}(x)=\frac{2}{\pi} \arctan (x / \delta) x>0$, with the p.d.f.

$$
g(x)=\frac{2}{\pi \delta}\left(1+\left(\frac{x}{\delta}\right)^{2}\right)^{-1}, \quad x>0,
$$

then (4) reduces to

$$
f(x)=\frac{a b 2^{a}}{\delta \pi^{a}}(\arctan (x / \delta))^{a-1}\left(1-(\arctan (x / \delta))^{a}\right)^{b-1}\left(1+(x / \delta)^{2}\right)^{-1}, x>0 .
$$

From (6), the c.d.f of the KW-Half-Cauchy can be written as

$$
F(x)=1-\left(1-\left(\frac{2}{\pi} \arctan (x / \delta)\right)^{a}\right)^{b}, x>0 .
$$

A random variable $X$ with the p.d.f $f(x)$ in (1.6) is said to follow the KW-Half-Cauchy distribution with parameters $\delta, a$ and $b$. When $a=1$ and $b=1$, then the KW-Half-Cauchy distribution reduces to the Half-Cauchy distribution with parameter $\delta$.

Again, using the generalized binomial expansion (1.3) can be written as 


$$
\begin{aligned}
F(x) & =\sum_{j=0}^{\infty}(-1)^{j}\left(\begin{array}{l}
b \\
j
\end{array}\right)\left(1-G^{a}(x)\right)^{j} \\
& =\sum_{j=0}^{\infty}(-1)^{j}\left(\begin{array}{l}
b \\
j
\end{array}\right)\left(\sum_{k=0}^{\infty}(-1)^{k}\left(\begin{array}{l}
a \\
k
\end{array}\right) G^{k}(x)\right)^{j} \\
& =\sum_{j=0}^{\infty} \sum_{k_{1}=0}^{\infty} \cdots \sum_{k_{j}=0}^{\infty}(-1)^{j+s_{k}} P_{k} G_{\delta}^{s_{k}}(x)
\end{aligned}
$$

where $s_{k}=k_{1}+k_{2}+\cdots+k_{j}$ and $P_{k}=\prod_{i=1}^{j}\left(\begin{array}{c}a \\ k_{i}\end{array}\right)$. By simple differentiation, it is immediate from (1.8),

$$
f(x)=\sum_{j=0}^{\infty} \sum_{k_{1}=0}^{\infty} \cdots \sum_{k_{j}=0}^{\infty}(-1)^{j+s_{k}} P_{k} s_{k} G^{s_{k}-1} \delta(x) g(x) .
$$

Next, using the expansion $\arctan x=\sum_{i=0}^{\infty} a_{i} \frac{x^{2 i+1}}{\left(1+x^{2}\right)^{i+1}}$, where $a_{i}=\frac{2^{2 i}(i !)^{2}}{(2 i+1) !}, G_{\delta}(x)$ can be written as $G_{\delta}(x)=\frac{x}{\delta^{2}+x^{2}} \sum_{i=0}^{\infty} b_{i}\left(\frac{x^{2}}{\delta^{2}+x^{2}}\right)^{i}$, where $b_{i}=\frac{2 \delta a_{i}}{\pi}$. By application of an equation from Gradshteyn and Ryznik (2007) for a power series raised to a positive integer $s_{k}$, we obtain

$$
G_{\delta}^{s_{k}}(x)=\left(\frac{x}{\delta^{2}+x^{2}}\right)^{s_{k}} \sum_{i=0}^{\infty} c_{s_{k}, i}\left(\frac{x^{2}}{\delta^{2}+x^{2}}\right)^{i}
$$

where the coefficients $c_{s_{k}, i}$, for $i=1,2, \cdots$ can be determined from the recursive equation $c_{s_{k}, i}=$ $\left(i b_{0}\right)^{-1} \sum_{m=1}^{i}\left[\left(s_{k}+1\right) m-i\right] b_{m} c_{s_{k}, i-m}$. The coefficients $c_{s_{k}, i}$ follows recursively $c_{s_{k}, 0}, \cdots, c_{s_{k}, i-1}$ and then from $b_{0}, \cdots, b_{i}$. So, we can rewrite (1.9) as

$$
f(x)=\sum_{j=0}^{\infty} \sum_{k_{1}=0}^{\infty} \cdots \sum_{k_{j}=0}^{\infty}(-1)^{j+s_{k}} P_{k} s_{k} c_{s_{k}, i} \frac{x^{s_{k}+2 i-1}}{\left(\delta^{2}+x^{2}\right)^{s_{k}+i}}, x>0 .
$$

\section{Properties of the KW-Half-Cauchy distribution}

We provide below a characterization of the KW-Half-Cauchy distribution which establishes the relation between KW-Half-Cauch and uniform distribution.

Lemma 1.(Transformation): If a random variable $U$ follows a uniform $(0,1)$ distribution with parameters then $X=\delta \tan \left(\frac{\pi}{2}\left(1-\left(1-(1-U)^{1 / b}\right)^{1 / a}\right)\right)$ follows the KW-Half-Cauchy with parameters $\delta, a$ and $b$.

Proof. The result follows immediately using the transformation technique.

The hazard function associated with the KW-Half-Cauchy distribution is

$$
h_{f}(x)=\frac{g(x)}{1-G(x)}=\frac{\frac{a b 2^{a}}{\delta \pi^{a}}(\arctan (x / \delta))^{a-1}\left(1+(x / \delta)^{2}\right)^{-1}}{\left(1-\left(\frac{2}{\pi} \arctan (x / \delta)\right)^{a}\right)^{b}}, x>0 .
$$


The limiting behaviors of the KW-Half-Cauchy and the hazard function are given in the following theorem.

Theorem 1. The limit of the KW-Half-Cauchy density function and the hazard function is given by

$$
\begin{gathered}
\lim _{x \rightarrow 0^{+}} f(x)=\lim _{x \rightarrow 0^{+}} h_{f}(x)= \begin{cases}0, & a>1, b>1 \\
\infty, & \max (a, b)<1 .\end{cases} \\
\lim _{x \rightarrow \infty} f(x)=\lim _{x \rightarrow \infty} h_{f}(x)= \begin{cases}0, & b>1 \\
\infty, & \max (a, b)<1 .\end{cases}
\end{gathered}
$$

Proof. Straightforward and hence omitted.

In Figures 1 and 2, various graphs of $g(x)$, and $h_{g}(x)$ are provided for different parameter values. The plots indicate that the KW-Half-Cauchy can be approximately symmetric, right-skewed or leftskewed. Also, the KW-Half-Cauchy hazard function can be a decreasing failure rate or upside down bathtub shapes.
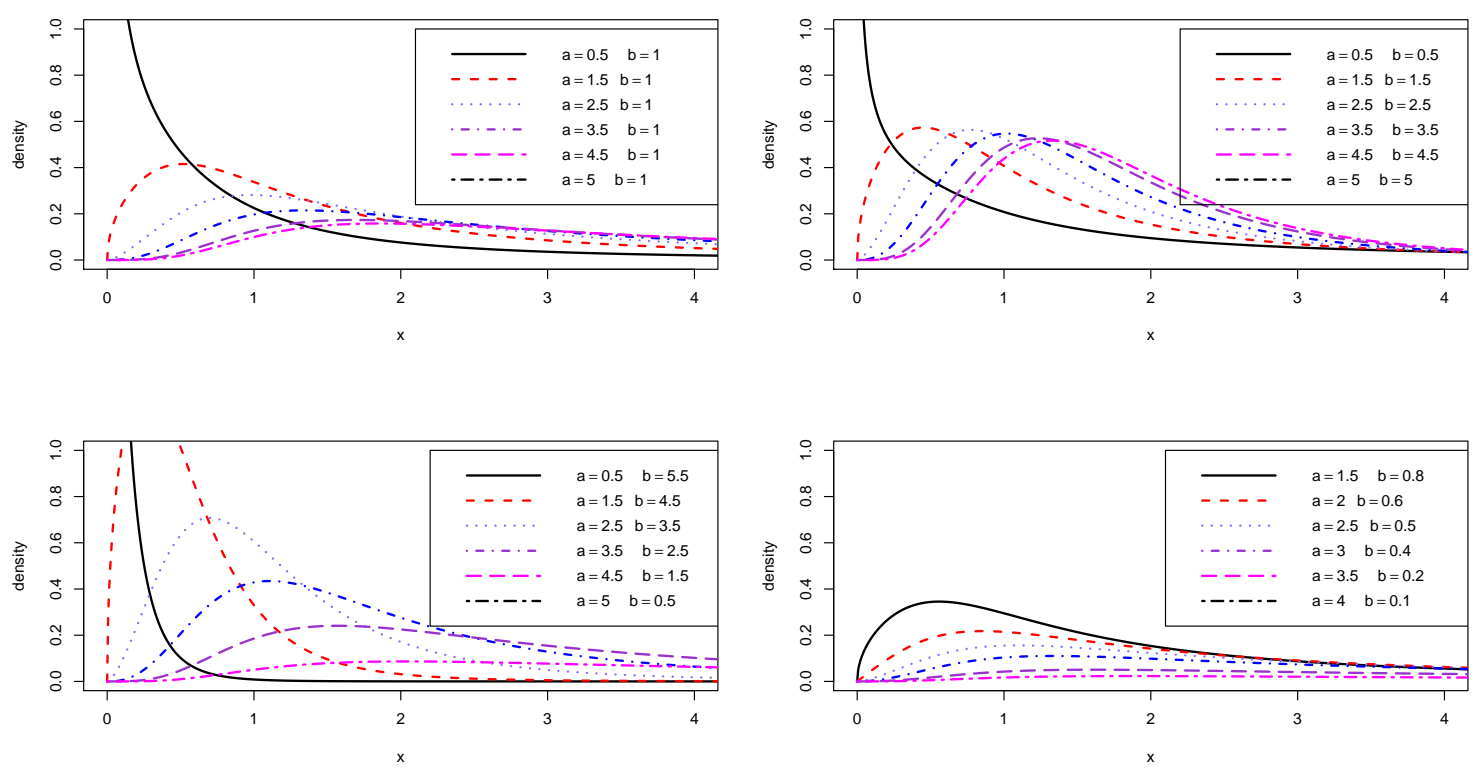

Fig. 1. Graphs of the KW-HC p.d.f for various choices of $\delta, a$ and $b$.

Let $X$ and $Y$ be two random variables. $X$ is said to be stochastically greater than or equal to $Y$, denoted by $X \geqslant Y$ if $P(X>x) \geq P(Y>x)$ for all $x$ in the support set of $X$.

Theorem 2. Suppose $X \sim \mathrm{KW}-\mathrm{HC}\left(\delta_{1}, a_{1}, b_{1}\right)$ and $Y \sim \mathrm{KW}-\mathrm{HC}\left(\delta_{2}, a_{2}, b_{2}\right)$. If $\delta_{1}>\delta_{2}, a_{1}<a_{2}$ and $b_{1}>b_{2}$ Then $X \geqslant Y$. 

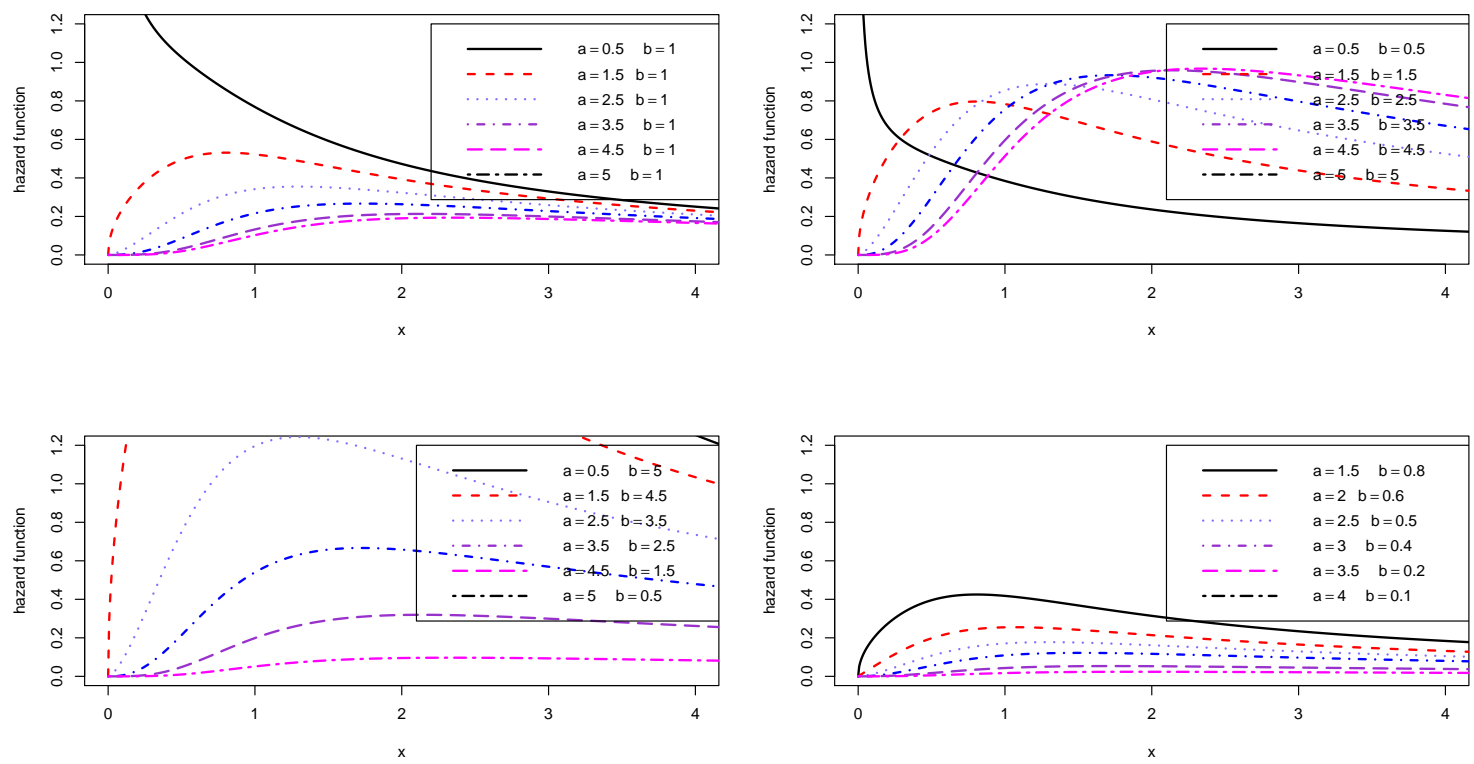

Fig. 2. Graphs of the KW-HC hazard function for various choices of $\delta, a$ and $b$.

Proof. For any real number $x \in \mathbb{R}^{+}, \alpha_{1}>\alpha_{2}, \delta_{1}>\delta_{2}, a_{1}<a_{2}$ and $b_{1}>b_{2}$, we have

$$
\left(1-\left(\frac{2}{\pi} \arctan \left(x / \delta_{1}\right)\right)^{a_{1}}\right)^{b_{1}} \geq\left(1-\left(\frac{2}{\pi} \arctan \left(x / \delta_{1}\right)\right)^{a_{2}}\right)^{b_{2}},
$$

which implies that $P(X>x) \geq P(Y>x)$ and this completes the proof.

The asymptotes of Equations (1.6) and (1.11) are given by

- As $x \rightarrow 0, f(x) \sim \frac{a b 2^{a}}{\delta \pi^{a}}(\arctan (x / \delta))^{a-1}\left(1+(x / \delta)^{2}\right)^{-1}$.

- As $x \rightarrow \infty, f(x) \sim \frac{a b 2}{\delta \pi}\left(1+(x / \delta)^{2}\right)^{-1}\left(1-\left(\frac{2}{\pi} \arctan (x / \delta)\right)^{a}\right)^{b-1}$.

- As $x \rightarrow 0, h_{f}(x) \sim \frac{a b 2^{a}}{\delta \pi^{a}}(\arctan (x / \delta))^{a-1}\left(1+(x / \delta)^{2}\right)^{-1}$.

- As $x \rightarrow \infty, h_{f}(x) \sim \frac{\frac{a b 2}{\delta \pi}\left(1+(x / \delta)^{2}\right)^{-1}}{1-\left(\frac{2}{\pi} \arctan (x / \delta)\right)^{a}}$.

\subsection{Simulation}

We present two different methods for simulation from the KW-Half-Cauchy distribution. The first method uses the inversion method. The quantile function corresponding to the density in (4) is given by

$$
\xi=F^{-1}(U)=\delta \tan \left(\frac{\pi}{2}\left(1-(1-U)^{1 / b}\right)^{1 / a}\right),
$$

where $U$ is a uniform variate on the unit interval $[0,1]$. 
The next method for simulation from the KW-Half-Cauchy distribution is based on the rejection method. The rejection method is based upon a theorem (see Arnold et al. (1999)) that assumes that the density of $X$ can be written in the following form:

$$
f(x)=C g(x) h(x),
$$

where $C>1,0<h(x)<1$ and $g(x)$ is also a probability density function. This method will be applicable in our case if $a \geq 1$ and $b \geq 1$. Define a constant $C$ by $C=\frac{a^{b} b(a-1)^{1-1 / b}(b-1)^{b-1}}{(a b-1)^{b-1 / a}}$.

Then the following scheme holds for simulating KW-Half-Cauchy:

(1) generate $X=x$ from the pdf $g(x)$ in (1.5);

(2) generate $Y=U C g(x)$, where $U$ is a uniform variate on the unit interval $[0,1]$;

(3) accept $X=x$ as a KW-Half-Cauchy variate if $Y<f(x)$. If $Y>f(x)$ return to step 2.

Skewness and kurtosis of a distribution can be measured by $\beta_{1}=\mu_{3} / \sigma^{3}$ and $\beta_{2}=\mu_{4} / \sigma^{4}$, respectively. However the expression for the third and fourth moments of $\mathrm{KW}-\mathrm{HC}(\delta, a, b)$ are difficult to obtain. Since the quantile function of $\mathrm{KW}-\mathrm{HC}(\delta, a, b)$ are in closed form, alternatively we can define the measure of skewness and kurtosis based on quantile function. The Galton' skewness $S$ defined by Galton (1883) and the Moors' kurtosis $K$ defined by Moors (1988) are given by

$$
\begin{gathered}
S=\frac{Q(6 / 8)-2 Q(4 / 8)+Q(2 / 4)}{Q(6 / 8)-Q(2 / 8)} . \\
K=\frac{Q(7 / 8)-Q(5 / 8)+Q(3 / 8)-Q(1 / 8)}{Q(6 / 8)-Q(2 / 8)} .
\end{gathered}
$$

When the distribution is symmetric, $S=0$ and when the distribution is right (or left) skew, $S>0$ (or $S<0$ ). As $K$ increases the tail of the distribution becomes heavier. To investigate the effect of the two shape parameters $\alpha$ and $\lambda$ on the $\mathrm{KW}-\mathrm{HC}(\delta, a, b)$ distribution, equation (2.5) and (2.6) are used to obtain the Galtons' skewness and Moors' kurtosis where the quantile function is defined in (2.4). Figure 3 displays the Galton's skewness and Moors' kurtosis for the KW-Half-Cauchy. From Figure 3, the KW-Half-Cauchy distribution can be left skewed, right skewed and symmetric.

\section{Moments and Mean deviations}

\subsection{Moments}

The moment of $X \sim \mathrm{KW}-\mathrm{HC}(\delta, a, b)$ can be expressed as

$$
E\left(X^{r}\right)=\sum_{j=0}^{\infty} \sum_{k_{1}=0}^{\infty} \cdots \sum_{k_{j}=0}^{\infty}(-1)^{j+s_{k}} P_{k} s_{k} c_{s_{k}, i} \cdot \int_{0}^{\infty} \frac{x^{s_{k}+2 i+r-1}}{\left(\delta^{2}+x^{2}\right)^{s_{k}+i}} d x
$$

For $0<\alpha<2 \rho$, the above integral can be calculated from Prudnikov et al. (1986, vol 1 , page 309, integral 7) as $\int_{0}^{\infty} \frac{x^{\alpha-1}}{\left(c^{2}+x^{2}\right)^{\rho}} d x=c^{\alpha-2 \rho} B(\alpha, 2 \rho-\alpha)_{2} F_{1}(\alpha / 2, \rho-\alpha / 2 ; \rho+1 / 2 ; 1)$, where ${ }_{2} F_{1}(p, q ; c ; z)=\sum_{j=0}^{\infty} \frac{(p)_{j}(q)_{j} z^{j}}{(c)_{j} j !}$ is the hypergeometric function and $(p)_{i}=p(p+1) \cdots(p+i-1)$ is 

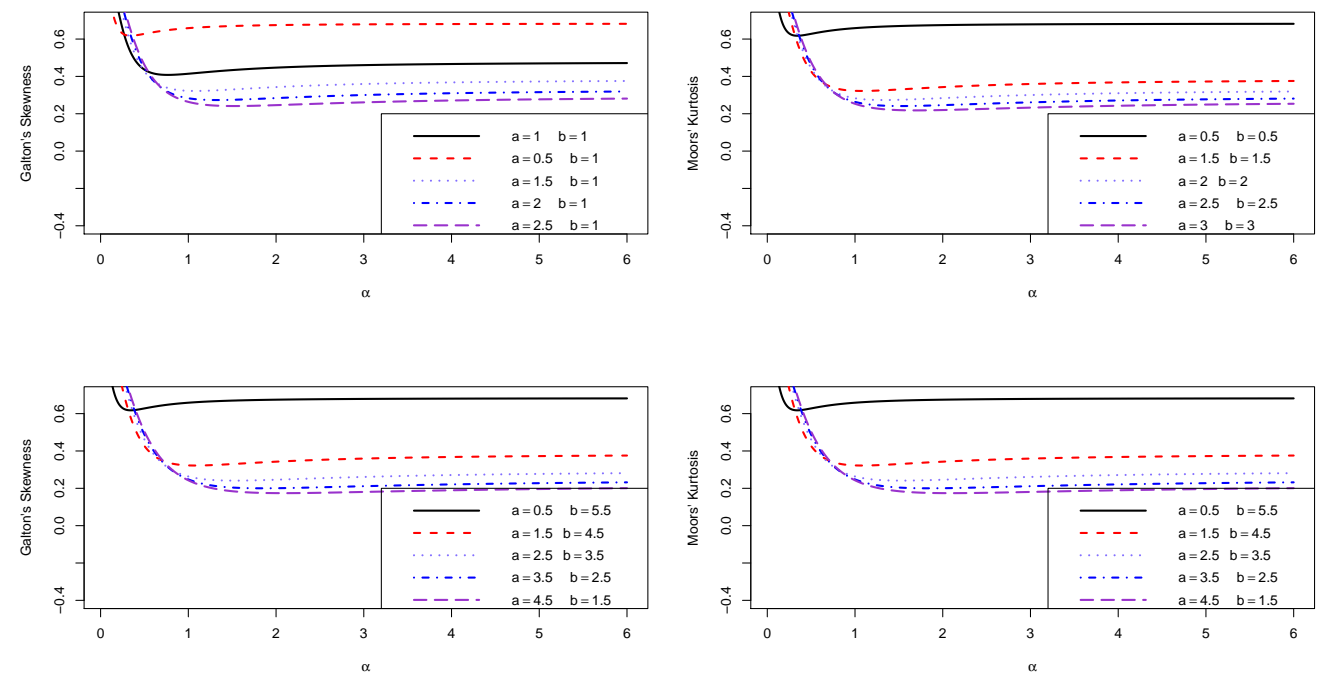

Fig. 3. Graphs of Skewness and Kurtosis for the KW-Half-Cauchy for different choices of $\delta, a$ and $b$.

the ascending factorial. Hence, for positive integer $a$ and $r<a$, one can get the following expression for the $r$-th order raw moments of $X$ which is as follows:

$$
\begin{aligned}
E\left(X^{r}\right)= & \sum_{j=0}^{\infty} \sum_{k_{1}=0}^{\infty} \cdots \sum_{k_{j}=0}^{\infty} \sum_{i=0}^{\infty}(-1)^{j+s_{k}} P_{k} s_{k} c_{s_{k}, i} \delta^{r-s_{k}} \\
& \times B\left(r+s_{k}+2 i, s_{k}-r\right){ }_{2} F_{1}\left(\frac{s_{k}+r}{2}+i, \frac{s_{k}-r}{2} ; s_{k} 2 i+1 / 2 ; 1\right) .
\end{aligned}
$$

\subsection{Mean deviations}

One can derive the KW-Half-Cauchy mean deviations about the mean $\mu=E(X)$ and the median $M=F^{-1}(1 / 2)$ using the relations $\xi_{1}=2 \mu F(\mu)-2 H(\mu)$ and $\xi_{2}=E(X)-2 H(M)$, where $\mu$ can be computed from (19), with $r=1$, and

$$
\begin{aligned}
H(r) & =\int_{0}^{s} x f(x) d x \\
& =\int_{0}^{F(s)}(Q(u))^{s} d u \\
& =\int_{0}^{F(s)}\left(\delta \tan \left(\frac{\pi}{2}\left(1-(1-u)^{1 / b}\right)^{1 / a}\right)\right)^{s} d u .
\end{aligned}
$$

Next, on using the expression $\tan x=\sum_{n=0}^{\infty} \frac{B_{2 n}(-4)^{n}\left(1-4^{n}\right)}{2 n !} x^{2 n-1}, \quad|x|<\pi / 2$, where $B_{k}$ 's are Bernoulli numbers, we get from (3.3)

$$
H(r)=\delta^{s}(\pi / 2)^{s} \sum_{n_{1}=0}^{\infty} \cdots \sum_{n_{s}=0}^{\infty} A_{s} \int_{0}^{F(s)}\left(1-(1-u)^{1 / b}\right)^{s / a} d u
$$


where $A_{s}=\prod_{k=1}^{s} \frac{B_{2 n_{k}}(-4)^{n_{k}}\left(1-4^{n_{k}}\right)}{2^{k} n_{k} !}$. Next, consider the integral

$$
\begin{aligned}
\int_{0}^{F(s)}\left(1-(1-u)^{1 / b}\right)^{s / a} d u & =\sum_{j=0}^{\infty}(-1)^{j}\left(\begin{array}{c}
(s / a) \\
j
\end{array}\right) \int_{0}^{F(s)}(1-u)^{j / b} d u \\
& =\sum_{j=0}^{\infty}(-1)^{j}\left(\begin{array}{c}
(s / a) \\
j
\end{array}\right) \frac{1-(1-F(s))^{j / b+1}}{j / b+1},
\end{aligned}
$$

if $s / a$ is an integer, then the summation in (3.5) will stop at $s / a$.

Hence, on substitution (3.5) in (3.4),

$$
H(r)=\delta^{s}(\pi / 2)^{s} \sum_{n_{1}=0}^{\infty} \cdots \sum_{n_{s}=0}^{\infty} \sum_{j=0}^{\infty}(-1)^{j} A_{s}\left(\begin{array}{c}
(s / a) \\
j
\end{array}\right) \frac{1-(1-F(s))^{j / b+1}}{j / b+1} .
$$

An application of the mean deviations is to the Lorenz and Bonferroni curves that are important in fields like economics, reliability, demography, insurance and medicine. They are defined for a given probability $\psi$ by $L(\psi)=\frac{H(q)}{\mu}$ and $B(\psi)=\frac{H(q)}{\pi \mu}$, respectively, where $q=Q(\psi)=$ $\delta \tan \left(\frac{\pi}{2}\left(1-(1-\psi)^{1 / b}\right)^{1 / a}\right)$. In economics, if $\psi=F(q)$ is the proportion of units whose income is lower than or equal to $q, L(\psi)$ gives the proportion of total income volume accumulated by the set of units with an income lower than or equal to $q$. On substitution $\psi$ in (3.6), one can get an expression for $L(\psi)$.

\section{Reliability parameter}

The reliability parameter $R$ is defined as $R=P(Y>X)$, where $X$ and $Y$ are independent random variables. Numerous applications of the reliability parameter have appeared in the literature such as the area of classical stress-strength model and the break down of a system having two components. Other applications of the reliability parameter can be found in Hall (1984) and Weerahandi and Johnson (1992).

If $X$ and $Y$ are two continuous and independent random variables with the c.d.f's $F_{1}(x)$ and $F_{2}(y)$ and their p.d.fs $f_{1}(x)$ and $f_{2}(y)$ respectively, then the reliability parameter $R$ can be written as

$$
R=P(Y>X)=\int_{-\infty}^{\infty} F_{1}(t) f_{2}(t) d t
$$

Theorem 3. Suppose that $X \sim K W-H C\left(\delta, a_{1}, b_{1}\right)$ and $Y \sim K W-H C\left(\delta, a_{2}, b_{2}\right)$, then

$$
R=\sum_{j=0}^{\infty}(-1)^{j}\left(\begin{array}{c}
b_{1} \\
j
\end{array}\right) B\left(j \frac{a_{1}}{a_{2}}+1, b_{2}\right) .
$$

Proof: From (1.6) and (1.7), we have

$$
\begin{aligned}
P(Y>X)= & \int_{0}^{\infty}\left(1-\left(\frac{2}{\pi} \arctan (y / \delta)\right)^{a_{1}}\right)^{b_{1}} \frac{a_{2} b_{2} 2^{a_{2}}}{\delta \pi^{a_{2}}}(\arctan (y / \delta))^{a_{2}-1} \\
& \times\left(1-(\arctan (y / \delta))^{a_{2}}\right)^{b_{2}-1}\left(1+(y / \delta)^{2}\right)^{-1} .
\end{aligned}
$$

On using the substitution $(\arctan (y / \delta))^{a_{2}}=u,(4.1)$, reduces to 


$$
\begin{aligned}
P(Y>X) & =b_{2} \int_{0}^{1}\left(1-u^{a_{1} / a_{2}}\right)^{b_{1}}(1-u)^{b_{2}-1} \\
& =\sum_{j=0}^{\infty}(-1)^{j}\left(\begin{array}{c}
b_{1} \\
j
\end{array}\right) B\left(j \frac{a_{1}}{a_{2}}+1, b_{2}\right),
\end{aligned}
$$

on using the generalized binomial expansion of $\left(1-u^{a_{1} / a_{2}}\right)^{b_{1}}=\sum_{j=0}^{\infty}(-1)^{j}\left(\begin{array}{c}b_{1} \\ j\end{array}\right) u^{j \frac{a_{1}}{a_{2}}}$. Note that if $b_{1}$ is an integer, then the above summation will stop at $b_{1}$.

\section{Order statistics}

The density function of the $r$-th order statistic is given by

$$
\begin{aligned}
& f_{X_{r: n}}(x) \\
& =\frac{g(x)}{B(r, n-r+1)} \sum_{l=0}^{\infty}(-1)^{l}\left(\begin{array}{c}
n-r \\
l
\end{array}\right)(G(x))^{r+l-1} \\
& =(B(r, n-r+1))^{-1} \sum_{j=0}^{\infty} \sum_{k_{1}=0}^{\infty} \cdots \sum_{k_{j}=0}^{\infty} \sum_{l=0}^{\infty}(-1)^{j+l+s_{k}} P_{k} s_{k} c_{s_{k}+r+l, i}\left(\begin{array}{c}
n-r \\
l
\end{array}\right) \frac{x^{s_{k}+r+l+2 i-1}}{\left(\boldsymbol{\delta}^{2}+x^{2}\right)^{s_{k}+r+l+i}}
\end{aligned}
$$

using (1.11).

Hence, the $m$-th order raw moments (for $m \geq 1$ ) will be

$$
\begin{aligned}
& E\left(X_{r: n}^{m}\right) \\
& =(B(r, n-r+1))^{-1} \sum_{j=0}^{\infty} \sum_{k_{1}=0}^{\infty} \cdots \sum_{k_{j}=0}^{\infty} \sum_{l=0}^{\infty}(-1)^{j+l+s_{k}} P_{k} s_{k} c_{s_{k}+r+l, i}\left(\begin{array}{c}
n-r \\
l
\end{array}\right) \delta^{m-\left(s_{k}+r+l\right)} \\
& \times B\left(m+s_{k}+r+l+2 i, s_{k}+r+l-m\right){ }_{2} F_{1}\left(\frac{s_{k}+r+l+m}{2}+i, \frac{s_{k}+r+l-m}{2} ; s_{k}+r+l+m+i+1 / 2 ; 1\right) .
\end{aligned}
$$

The $L$-moments are summary statistics for probability distributions and data samples (Hosking, 1990). It has the advantage that it exists whenever the mean of the distribution exists, although some higher moments may not exist. It can be expressed as a linear combination of the ordered data values $\phi_{r}=\sum_{j=0}^{r-1}(-1)^{r-1-j}\left(\begin{array}{c}r-1 \\ j\end{array}\right)\left(\begin{array}{c}r-1+j \\ j\end{array}\right) \eta_{j}$, where $\eta_{j}=E\left(X F^{j}(X)\right)=(j+1)^{-1} E\left(X_{j+1, j+1}\right)$. In particular $\phi_{1}=\eta_{0}, \phi_{2}=2 \eta_{1}-\eta_{0}$ and $\phi_{3}=6 \eta_{2}-6 \eta_{1}+\eta_{0}$. The $L$-moments of the KW-HalfCauchy distribution can be obtained from (26).

\subsection{Entropy}

The entropy of a random variable $X$ with the density function $f(x)$ is a measure of variation of the uncertainty. Renyi entropy is defined as $I_{R}(\rho)=(1-\rho)^{-1} \log \left(\int f^{\rho}(x) d x\right)$, where $\rho>0$ and $\rho \neq 1$. If a random variable $X$ has a KW-HC distribution, then, we have, 


$$
\begin{aligned}
& f^{\rho}(x) \\
& =\left(\frac{a b 2^{a}}{\delta \pi^{a}}\left(1+(x / \delta)^{2}\right)^{-1}\right)^{\rho} \sum_{j=0}^{\infty}\left(\begin{array}{c}
(b-1) \rho \\
j
\end{array}\right) G^{(a-1) \rho+a j}(x) \\
& =\left(\frac{a b 2^{a}}{\delta \pi^{a}}\left(1+(x / \delta)^{2}\right)^{-1}\right)^{\rho} \sum_{j=0}^{\infty}\left(\begin{array}{c}
(b-1) \rho \\
j
\end{array}\right)\left(\left(\frac{x}{\delta^{2}+x^{2}}\right)^{a(j+\rho)-\rho} \sum_{i=0}^{\infty} c_{a(j+\rho)-\rho, i}\left(\frac{x^{2}}{\delta^{2}+x^{2}}\right)^{i}\right) \\
& =\left(\frac{a b \delta^{2} 2^{a}}{\delta \pi^{a}}\right)^{\rho} \times \sum_{j=0}^{\infty} \sum_{i=0}^{\infty}\left(\begin{array}{c}
(b-1) \rho \\
j
\end{array}\right) c_{a(j+\rho)-\rho, i}\left(\frac{x^{a(j+\rho)-\rho+2 i}}{\left(\delta^{2}+x^{2}\right)^{a(j+\rho)+i}}\right)
\end{aligned}
$$

Now, by Prudnikov et al. (1986), the following integral can be evaluated as

$$
\begin{aligned}
\int_{0}^{\infty} \frac{x^{a(j+\rho)-\rho+2 i}}{\left(\delta^{2}+x^{2}\right)^{a(j+\rho)+i}=} & \delta^{i+1-\rho} B(a(j+\rho)-\rho+2 i+1, \rho(a+1)+a j-1) \\
& \times{ }_{2} F_{1}\left(i+\frac{a(j+\rho)-\rho+1}{2}+i, \frac{a(j+\rho)}{2}-\frac{\rho-2 i-1}{2} ; a(j+\rho)+i+1 / 2 ; 1\right) .
\end{aligned}
$$

Hence, the expression for the Renyi entropy in this case will be

$$
\begin{aligned}
I_{R}(\rho)= & \left(\frac{a b \delta^{i+1+\rho} 2^{a}}{\delta \pi^{a}}\right)^{\rho} \times \sum_{j=0}^{\infty} \sum_{i=0}^{\infty}\left(\begin{array}{c}
(b-1) \rho \\
j
\end{array}\right) c_{a(j+\rho)-\rho, i} B(a(j+\rho)-\rho+2 i+1, \rho(a+1)+a j-1) \\
& \times{ }_{2} F_{1}\left(i+\frac{a(j+\rho)-\rho+1}{2}+i, \frac{a(j+\rho)}{2}-\frac{\rho-2 i-1}{2} ; a(j+\rho)+i+1 / 2 ; 1\right) .
\end{aligned}
$$

\section{Maximum likelihood estimation}

Let $X_{1}, X_{2}, \cdots, X_{n}$ be a random sample of size $n$ drawn from the density in (1.6). The log-likelihood function is given by

$$
\begin{aligned}
\ell & =n \log (a b)+n a \log (2 / \pi)-n \log \delta+(b-1) \sum_{i=1}^{n} \log \left(1-\left(2 / \pi \arctan \left(x_{i} / \delta\right)\right)^{a}\right) \\
& +(a-1) \sum_{i=1}^{n} \arctan \left(x_{i} / \delta\right)-\sum_{i=1}^{n} \log \left(1+\left(x_{i} / \delta\right)^{2}\right)^{-1} .
\end{aligned}
$$

The derivatives of (6.1) with respect to $a, b$, and $\delta$ are given by

$$
\begin{gathered}
\frac{\partial}{\partial a} \ell=\frac{n}{a}+n \log (2 / \pi)+\sum_{i=1}^{n} \arctan \left(x_{i} / \delta\right)-(b-1) \sum_{i=1}^{n}\left(1-\left(2 / \pi \arctan \left(x_{i} / \delta\right)\right)^{a}\right)^{-1} \\
\times\left(2 / \pi \arctan \left(x_{i} / \delta\right)\right)^{a} \log \left(2 / \pi \arctan \left(x_{i} / \delta\right)\right) . \\
\frac{\partial}{\partial b} \ell=\frac{n}{b}+\sum_{i=1}^{n} \log \left(1-\left(2 / \pi \arctan \left(x_{i} / \delta\right)\right)^{a}\right) .
\end{gathered}
$$




$$
\begin{aligned}
\frac{\partial}{\partial \delta} \ell= & a(b-1) \sum_{i=1}^{n}\left(1-\left(2 / \pi \arctan \left(x_{i} / \delta\right)\right)^{a}\right)^{-1}\left(2 / \pi \arctan \left(x_{i} / \delta\right)\right)^{a-1} \frac{2}{\pi}\left(1+\left(x_{i} / \delta\right)^{2}\right)^{-1}\left(x_{i} / \delta^{2}\right) \\
& +(2-a) \sum_{i=1}^{n}\left(x_{i} / \delta^{2}\right)\left(1+\left(x_{i} / \delta\right)^{2}\right)^{-1} .
\end{aligned}
$$

The MLE $\hat{a}, \hat{b}$, and $\hat{\delta}$ are obtained by setting (6.2), (6.3) and (6.4) to zero and solving them simultaneously.

Since KW-HC distribution reduces to HC distribution when $a=1 \quad b=1$, one can use the likelihood ratio test to compare if the $\mathrm{KW}-\mathrm{HC}$ distribution is a better choice for fitting a given data set than the HC distribution. The likelihood test that tests the hypothesis $H_{0}: a=1, \quad b=1$ against $H_{a}: a, b \neq 1$ is based on $\lambda=L_{0}(\tilde{\delta}) / L_{a}(\hat{\delta}, \hat{a}, \hat{b})$, where $L_{0}$ and $L_{a}$ are the likelihood functions for the $\mathrm{HC}$ and the KW-HC distributions respectively. In this case the quantity $-2 \log \lambda$ follows the chi-square distribution with 2 degree of freedom asymptotically.

\section{Application}

In this section, one data set is fitted to the KW-HC distribution. The data set in Table 1 resulted from tests on the endurance of deep-groove ball bearings and they represent the number of millions revolutions reached by each bearing before fatigue failure (Lieblein and Zelen, 1956). The KW-HC distribution is fitted to the data set in Table 1 and compared the result with the Half-Cauchy (HC), Beta-Half-Cauchy (BHC, Cordeiro et al., 2011), and Exponentiated-Half-Cauchy(EHC). These results are reported in Table 2. The results show that BHC, EHC, and KW-HC distributions provide adequate fit to the data. Figure 4 displays the empirical and the fitted cumulative distribution functions. This figure supports the results in Table 2. A close look at Figure 4 indicates that the KW$\mathrm{HC}$ provides better fit to the left tail than the BHC distributions. This due to the fact that KW-HC can have longer left tail (see Figure 1).

Table 1. Endurance of deep-groove ball bearings data.

\begin{tabular}{|cccccc|}
\hline 17.88 & 45.60 & 54.12 & 68.88 & 105.84 & 28.92 \\
48.40 & 55.56 & 84.12 & 127.92 & 33.00 & 51.84 \\
67.80 & 93.12 & 128.04 & 41.52 & 51.96 & 68.64 \\
98.64 & 173.40 & 42.12 & 54.12 & 68.64 & 105.12 \\
\hline
\end{tabular}

\section{Conclusion}

A special case of the Kumaraswamy- $G$ family of distributions, the Kumaraswamy-Half-Cauchy distribution is defined and studied. Various properties of the Kumaraswamy-Half-Cauchy distribution are investigated, including moments, hazard function, reliability parameter. The new model includes as special sub-models the Half-Cauchy and the exponentiated Half-Cauchy. It is observed that the distribution can be symmetric, positively skewed and negatively skewed with a broader class of monotone hazard rates. An application to a real data set shows that the fit of the new model is superior to the fits of its main sub-models. Estimation of the model parameters under the bayesian paradigm is currently underway and will be reported in a separate article elsewhere. 
Table 2. Parameter estimates for endurance of deep-groove ball bearings data.

\begin{tabular}{|c|c|c|c|c|}
\hline Distribution & $\mathrm{HC}$ & BHC & EHC & KW-HC \\
\hline Parameter Estimates & $\hat{\delta}=0.0285$ & $\begin{array}{c}\hat{\delta}=1.2285 \\
\hat{a}=12.9784 \\
\hat{b}=2.3629\end{array}$ & $\begin{array}{c}\hat{\delta}=6.674 \\
\hat{a}=4.1837 \\
\hat{b}=3.1825\end{array}$ & $\begin{array}{l}\hat{\delta}=5.0249 \\
\hat{a}=4.1123 \\
\hat{b}=2.8709\end{array}$ \\
\hline Log likelihood & -161.39 & -146.85 & -182.93 & -109.25 \\
\hline AIC & 316.40 & 189.44 & 245.68 & 117.36 \\
\hline K-S & 0.5245 & 0.09597 & 0.1315 & 0.0417 \\
\hline K-S p-value & 0.0000 & 0.8740 & 0.7564 & 0.9986 \\
\hline
\end{tabular}

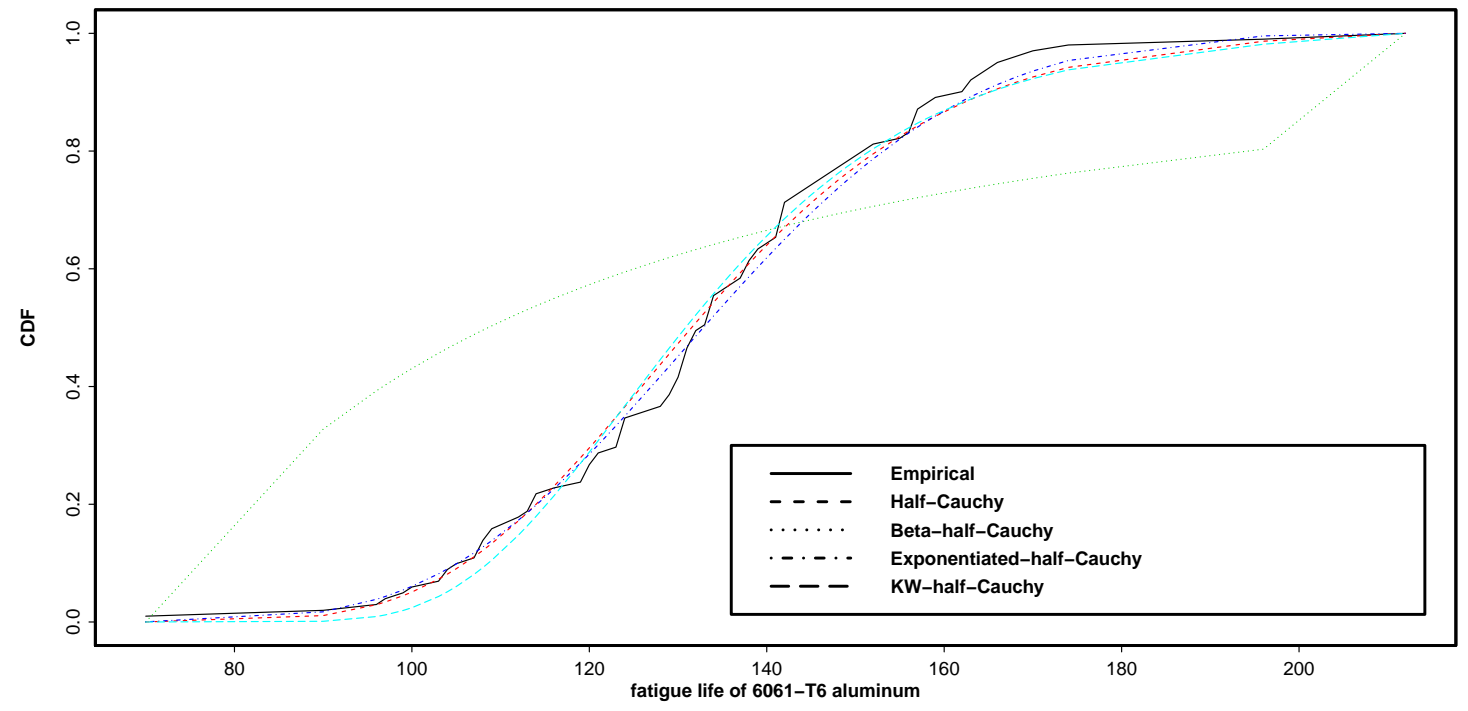

Fig. 4. CDF for fitted distributions of the endurance of deep-groove ball bearings data.

\section{Acknowledgement}

The author do not have a conflict of interests in this manuscript. 


\section{References}

[1] Arnold, B.C., Castillo, E., and Sarabia, J.M. (1999). Conditional Specification of Statistical Models. Springer-Verlag, New York.

[2] Cordeiro G. M. and de Castro, M. (2011). A new family of generalized distributions. Journal of Statistical Computation and Simulation, 81, 883898.

[3] Galton, F. (1883). Enquiries into Human Faculty and Its Development. London, Macmillan.

[4] Gradshteyn, I.S., Ryzhik, I.M. (2007). Table of Integrals, Series, and Products. Academic Press, New York.

[5] Hall, I.J. (1984). Approximate one-sided tolerance limits for the difference or sum of two independent normal variates. Journal of Qualitative Technology, 16,15-19.

[6] Jones, M. C. (2009). A beta-type distribution with some tractability advantages. Statistical Methodology, 6, 7081

[7] Kumaraswamy, P. (1980). Generalized probability density-function for double-bounded randomprocesses. Journal of Hydrology, 462, 7988.

[8] Lieblein, J. and Zelen, M. (1956). Statistical Investigation of the Fatigue Life of Deep-Groove Ball Bearings. Journal of Research of the National Bureau of Standards, 57, 273-316.

[9] Moors, J.J.A. (1988). A quantile alternative for Kurtosis. The Statistician, 37, 25-32.

[10] Nadarajah, S., Cordeiro, G.M. and Ortega, E.M.M. (2011). General results for the Kumaraswamy-G distribution. Journal of Statistical Computation and Simulation. DOI: 10.1080/00949655.2011.562504.

[11] Polson, N.G., and Scott, J.G. (2012). On the half-Cauchy prior for a global scale parameter. Bayesian Analysis 7, 887-902.

[12] Weerahandi, S. and Johnson, R.A. (1992). Testing reliability in a stress-strength model when X and Y are normally distributed. Technometrics, 38, 83-91. 